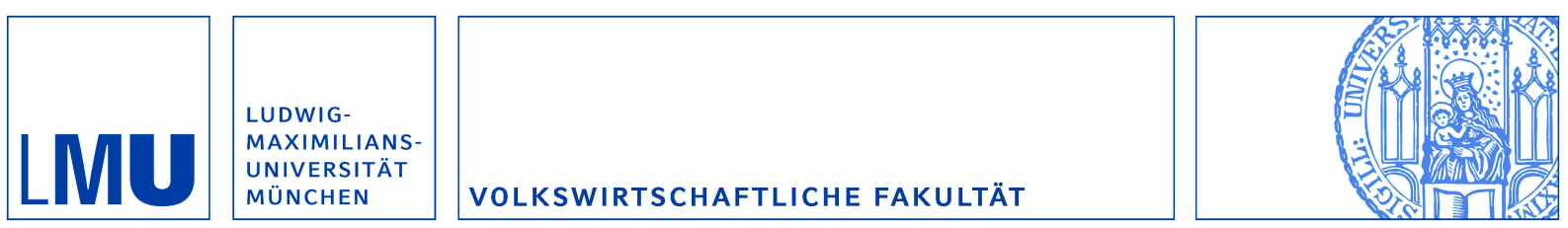

Lahno, Amrei M. und Lahno, Bernd:

Team Reasoning as a Guide to Coordination

Munich Discussion Paper No. 2014-8

Department of Economics

University of Munich

Volkswirtschaftliche Fakultät

Ludwig-Maximilians-Universität München

Online at https://doi.org/10.5282/ubm/epub.20822 


\title{
Team Reasoning as a Guide to Coordination
}

\author{
Amrei M. Lahno* \\ Department of Economics, University of Munich \\ Bernd Lahno\# \\ Legal Studies and Ethics Department, Frankfurt School of Finance \& Management
}

\section{This version: June 2014}

\begin{abstract}
A particular problem of traditional Rational Choice Theory is that it cannot explain equilibrium selection in simple coordination games. In this paper we analyze and discuss the solution concept for common coordination problems as incorporated in the theory of Team Reasoning (TR). Special consideration is given to TR's concept of opportunistic choice and to the resulting restrictions in using private information. We report results from a laboratory experiment in which teams were given a chance to coordinate on a particular pattern of behavior in a sequence of HiLo games. A modification of the stage game offered opportunities to improve on the team goal through changing this accustomed pattern of behavior. Our observations throw considerable doubt on the idea of opportunistic team reasoning as a guide to coordination. Contrary to what TR would predict, individuals tend to stick to accustomed behavioral patterns. Moreover, we find that individual decisions are at least partly determined by private information not accessible to all members of a team. Alternative theories of choice, in particular cognitive hierarchy theory may be more suitable to explain the observed pattern of behavior.
\end{abstract}

JEL codes: C91, C92, D03, D83, G02.

Keywords: team reasoning, collective agency, coordination, opportunistic choice, laboratory experiment.

Address and E-mail: *Geschwister-Scholl-Platz 1, D-80539 Munich, Germany; amrei.lahno@econ.lmu.de. \# Corresponding author; Sonnemannstraße 9-11, D-60314 Frankfurt am Main, Germany; b.lahno@fs.de.

We gratefully acknowledge funding from the Frankfurt School of Finance \& Management. Bernd Lahno's work on this project was supported by a fellowship at the Käte Hamburger Kolleg / Centre for Global Cooperation, University of Duisburg-Esssen, Germany. 


\section{Introduction}

It has always been transparent to the attentive observer that traditional rational choice theory (RC) performs poorly as a descriptive theory of human behavior in many areas of social interaction. In recent years, experiments with simple dilemma games by behavioral economists and other behavioral scientists validated this simple truth. The theory of team reasoning (TR; ?????) is one attempt to cope with this problem. A particular problem of traditional $\mathrm{RC}$ is that it cannot explain equilibrium selection in simple coordination games. Patterns of coordination very often seem evident from a common sense point of view, and, so, coordination is in fact rarely an actual problem for real individuals. Still, if actors were to ask RC for advice they would get utterly frustrated. RC remains silent if equilibrium selection is the issue. If individuals look at the problem from a team perspective, however, some of the most common routes to coordination become unveiled. If there is a single pattern of behavior that is best for the group (which often amounts to 'best for each individual in the group'), then it seems reasonable that each should do his ${ }^{1}$ part in the scheme of actions so defined. But a team perspective is not comprehensible within the classical rational choice approach to social interaction.

TR is an attempt to fill this gap by implementing team agency into the theory of rational action. Teams are added to the theory as possible agents, while the fundamental assumptions of $\mathrm{RC}$ about rational decision-making remain unchanged in principle. In TR, these assumptions are just transferred to the realm of the new agents and, thus, apply to individuals and groups alike. TR introduces team agency in two steps. First, team preferences are defined as a common scheme of evaluation. Second, a choice rule specifies how an individual is to contribute to maximizing team utility by making his (individual) choice.

Extending agency from individuals alone to individuals and groups seems in fact suitable to account for various instances of successful coordination and other cooperative regularities. Moreover, by reference to team agency, TR can explain the stability of behavioral patterns and behavioral expectations. It thus gives some account of social norms not available within RC. However, as TR sticks with the fundamental assumptions of instrumental rationality as conceptualized in RC it also inherits the idea of opportunistic choice. As individuals according to RC, teams according to TR instantly detect and take opportunities as soon as they arise. Choices of team members, therefore, are assumed to be principally unaffected by normative expectations or behavioral regularities observed in the past. They may conform with customs or norms to the extent that those actually

\footnotetext{
${ }^{1}$ We refer to a decision maker as "he" throughout the paper.
} 
promote the team interest. But neither customs nor norms constitute a fundamental restriction to decision-making.

An interesting consequence of opportunism as embodied in TR's choice rule is that individuals do not make use of the entire private information available to them. While the principles of individual opportunistic rationality would demand that each individual uses all the private information available to him, a team cannot make a decision dependent on information that is available to some but not all its members. As a result, the optimal scheme of action in terms of the team goal may be different depending on whether determined from an individual or from the team perspective.

In this paper we analyze and discuss the solution concept for common coordination problems as incorporated in TR. Special consideration is given to TR's concept of opportunistic choice and to the resulting restrictions in using private information. We report results from a laboratory experiment in which we analyze behavior in simple coordination dilemmas. In particular, we test whether 'teams' react opportunistically to changes in a strategic environment. ${ }^{2}$ In the experiment teams were given a chance to coordinate on a particular pattern of behavior in a sequence of HiLo games. Then, a modification of the stage game offered opportunities to improve on the team goal by changing this accustomed pattern of behavior. We implemented three treatments which differ with respect to the particular modification and the optimal strategy induced by team reasoning.

Our observations throw considerable doubt on the idea of opportunistic team reasoning as a guide to coordination. Contrary to what TR would predict, individuals tend to stick to accustomed behavioral patterns. Moreover, we find that individual decisions are at least partly determined by private information not accessible to all members of a team, which violates TR's assumption that individual solely use information which is available to all team members. Alternative theories of choice, in particular cognitive hierarchy theory (?), may be more suitable to explain the observed pattern of behavior.

The remainder of this paper is organized as follows. First, we briefly sketch the guiding ideas and basic elements of the theory of team reasoning. We illustrate the theory by discussing TR's suggestions to solve problems of cooperation as represented by the prisoners' dilemma and general problems of coordination as exemplified in a simple HiLo game (section 2). After specifying the empirical claims incorporated in TR as a descriptive theory (section 3) we investigate the theoretical relationship between $\mathrm{RC}$ and $\mathrm{TR}$ (section 4). We argue that TR inherits a strict conception of instrumental rationality and opportunistic choice from RC. To extract the opportunistic nature

\footnotetext{
${ }^{2}$ Experimental studies of the empirical validity of the theory of team reasoning seem to be rare. In an experimental comparison of TR and Cognitive Hierarchy, ? found mixed evidence for the explanative power of TR in coordination problems. ? found evidence for team reasoning in experiments with lifelike vignettes as well as in abstract games.
} 
of TR's concept of rational choice we consider variants of the HiLo game and derive behavioral predictions under different forms of uncertainty (section 5). We then test the empirical significance of TR by actually implementing these cooperation dilemmas in a laboratory experiment; we describe the experimental design, and present and discuss our results (section 6). Finally, we conclude and provide some general remarks (section 7 ).

\section{The foundations of team reasoning}

The core idea of TR is to abandon the assumption of exclusively individual agency in RC and amend traditional decision theory by an adequate account of collective agency. Consider a classic Prisoners' Dilemma (PD) as given in Figure 1 (payoffs normalized):

\begin{tabular}{|c|c|c|c|c|}
\hline & $\mathrm{C}$ & & D & \\
\hline$C$ & & $a$ & \begin{tabular}{l|l|}
0 & 1
\end{tabular} & \\
\hline & $d$ & 0 & 1 & 1 \\
\hline
\end{tabular}

Figure 1: Prisoners' dilemma

RC implies that rational individuals will mutually defect, $(\mathrm{D}, \mathrm{D})$, although both prefer mutual cooperation, (C, C). There is ample evidence that real decision makers in fact cooperate in such situations to a considerable extent. TR offers the following explanation: individuals do not always maximize their expected individual utility given what they believe about the actions of others as RC would demand. The individual actor may perceive a situation primarily as one that poses a problem to the group (the 'team') of the two actors as a whole rather then to each of them separately and in isolation. So, instead of asking What should I do?' the guiding question in the decision-making process is: 'What should we do?'. In a PD as given above the natural answer seems to be: 'We should cooperate!' meaning that the collective of the two actors should collectively realize the result (C, C). So both should do their part in the collective scheme to realize the collectively preferred result, each should cooperate. This decision-making procedure presupposes that there is a common scheme of evaluating the consequences of interaction from a team perspective. Given such a scheme, then, the question 'What should we do?' can be answered in the standard instrumental way by identifying the combination of actions that yields the best result according to team evaluation. TR prescribes that each individual does his part of the optimal scheme provided that it is common knowledge among the individuals that each individual identifies with the group and endorses the common evaluation scheme. Consequently, acting as a 'teamer' involves being guided by the team objective and a distinctive way of reasoning. TR therefore comprises two discernible parts: (1) a 
theory of preference transformation and (2) a decision-making rule.

It is, in fact, an extremely complex and difficult task to give a general account of how to derive team preferences from individual preferences and the relevant properties of a situation of choice. Nevertheless, in many situations we have quite firm intuitions about group evaluation. In our PD a plausible transformation from individual to team payoffs is given in Figure 2.

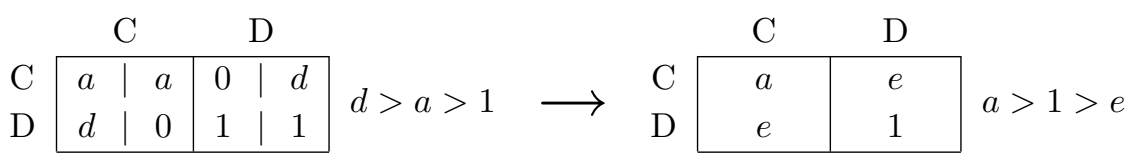

Figure 2: Team payoffs in a prisoners' dilemma

From the team perspective $(\mathrm{C}, \mathrm{C})$ is preferred to $(\mathrm{D}, \mathrm{D})$ and - in contrast to individual evaluation as given in the original matrix - also preferred to the asymmetrical outcomes (C, D) and (D, C). If all individuals take on the team perspective and if this is commonly known among them, team reasoners will proceed according to the following decision-making rule:

\section{Choose your part of the common scheme of actions that maximizes team payoff. (tr)}

One might wonder whether the choice rule of team reasoning as specified in (tr) may play a relevant role beyond traditional rules of individual rationality at all. ${ }^{3}$ Will it not suffice to assume that it is common knowledge that both players take on the team's objective? Will rational individuals in the traditional sense of RC, maximizing the so defined utility individually, not 'automatically' decide in exactly the same way as proposed by TR without the necessity to refer to a special decision rule? The answer is NO. Consider again the payoffs in the matrix to the right of Figure 2 and assume that the payoffs represent team as well as individual utilities derived from the respective action profiles. The payoff $e$ is assumed to be smaller than 1, which is a plausible assumption for team evaluation. According to standard $\mathrm{RC}$ this game has two equilibria, $(\mathrm{C}, \mathrm{C})$ and $(\mathrm{D}, \mathrm{D})$. As we shall argue in a moment, the resulting equilibrium selection problem cannot be solved on the basis of the standard assumptions of instrumental rationality alone. The choice rule (tr) solves this problem.

The problem of equilibrium selection in $\mathrm{RC}$ - for which $\mathrm{TR}$ offers a (partial) solution - is a general one. It is just as relevant as the notorious problem of cooperation in dilemma games but has not received the same sort of attention (as it deserves). Thus, taking a closer look is worthwhile.

\footnotetext{
${ }^{3}$ Although (tr) will inevitably produce cooperation in a PD it does not necessarily define an action for every individual in each and every strategic situation. If no unique optimal strategy profile exists (e.g., because of indifference in terms of team evaluation), then, obviously, an additional choice rule is needed to determine the scheme of actions to be collectively chosen.
} 
For illustration consider the following coordination game in Figure 3 (payoffs again normalized), which is known as the HiLo game.

\begin{tabular}{|c|c|c|c|c|}
\hline & rec & & blue & \\
\hline$e d$ & \begin{tabular}{l|l}
$a$ &
\end{tabular} & $a$ & \begin{tabular}{l|l}
$0 \quad 1$ &
\end{tabular} & \\
\hline ue & 0 & 0 & & 1 \\
\hline
\end{tabular}

Figure 3: HiLo game

Compared to the PD HiLo has an important property relevant to someone who is interested in the empirical validity of TR, namely, the preferences of both players are perfectly congruent. It is only natural to assume that team payoffs coincide with individual payoffs (see Figure 4) and the problem of deriving team preferences does not even evolve. ${ }^{4}$

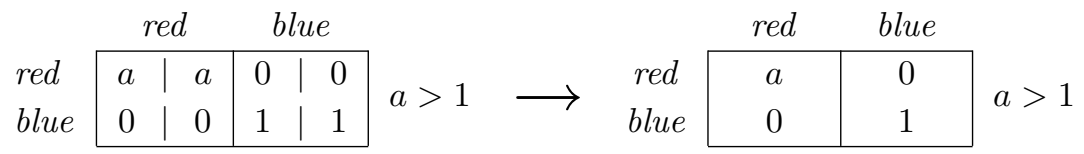

Figure 4: Team preferences in a HiLo game

Hence, HiLo is particularly suited to investigate the empirical validity of (tr), the team reasoning choice rule. We will concentrate on variants of this game in what follows. Consider, the HiLo in Figure 3 from a pure RC point of view. The game has two equilibria (red, red) and (blue, blue) with (red, red) dominating (blue, blue) in the Pareto sense. So red seems to be the unique rational choice in this situation (our experiment once more supports the conjecture that individuals consistently act in accordance with this simple insight). But notice that the Pareto-optimality of (red, red) is a property of a common scheme of action, which no individual can realize individually by his choice. ${ }^{5}$ Being solely based on conditions of individual rationality $\mathrm{RC}$ cannot substantiate reasons to choose red or blue in the following situation.

Consider an individual A playing against B. From an RC point of view A has reason to choose red only if he has reason to believe that B will also choose red (with sufficient probability). But B is in the same position. Knowing that B is rational like herself $\mathrm{A}$ therefore needs a reason to believe that B has reason to believe that A will choose red. But again: B is in the same position... Obviously this leads into an endless regress.

To assume that individual rationality as defined in $\mathrm{RC}$ might provide a decisive reason to act in certain ways - and, thus, provide a solution to the choice problem - in coordination problems

\footnotetext{
${ }^{4}$ Moreover, we may abstain from analyzing the role of (outcome-based) social preferences (see, e.g., ??). Obviously, concerns for distributions in outcomes do not influence utilities.

${ }^{5}$ The same is true of the property of being an equilibrium, and a similar argument applies (?).
} 
as HiLo is void. ${ }^{6}$ In contrast, TR provides an outright solution to the problem that fits very nicely with our intuitions. The fact that individual goals are completely congruent gives sufficient reason to assume that $\mathrm{A}$ and $\mathrm{B}$ realize their collective (joint) dilemma. (tr) is the core of this solution: the best result from everyone's point of view is brought about by mutually choosing red. And this is common knowledge. So, according to TR each has reason to follow (tr) and choose his part in the preferred scheme; each chooses red.

\section{Team reasoning as a descriptive theory}

TR is particularly relevant for situations in which choice may be conceived as an issue of a collective rather than of individuals. But even if a situation may be characterized as such from the outside, TR does not necessarily demand that individuals actually employ team reasoning. Team reasoning is conditional on a certain perception of the situation from inside, i.e., by the individuals that are to form the collective. It presupposes that individuals conceive themselves as members of a team and perceive this team as a possible unit of (collective) agency characterized by its team goals (cf. ?, 125). The essential element of such group identification then is that individuals take on the team goal as their own.

Whether or not an individual identifies with a team is a matter of framing, not a matter of rationality. Both Sugden and Bacharach agree that there are empirical regularities such that that group identification is promoted by certain situations but prevented by others. ${ }^{7}$ But these issues are understood as the object of empirical research in psychology or behavioral science, rather than as being an integral part of TR. Sugden takes TR - just like RC - as a theory without empirical content (?,203). Bacharach, however, identifies at least one condition of group identification within his account of TR. He, first, refers to theories of group identity and empirical research in psychology to present different states of affairs that may prompt group identification (?, $73 \mathrm{ff}$.). He, then, particularly identifies a source of group identification that is entirely determined by the abstract form of a situation as represented in a game. He refers to this source of group identification as "strong interdependence" $(?, 84)$. A situation is one of strong interdependence if there is a feasible outcome that Pareto-dominates every (other) possible solution consistent with the principles of individual rational decision-making as specified by RC. Note that the two abstract situations discussed above,

\footnotetext{
${ }^{6}$ See ? for a more detailed account of the problem. Different scholars have recognized and analyzed the problem; see ? for an overview on the 'classic' literature. Sugden traces the discovery and first analysis of the problem to ?.

${ }^{7}$ ? may serve to exemplify this idea. Based on the literature from scholars of the so called "social identity approach" in social psychology he argues that social categorization is a core factor in group identification.
} 
the PD as well as the HiLo, are of this sort. (C, C) Pareto-dominates (D, D), the unique solution according to $\mathrm{RC}$ in the $\mathrm{PD}$, and (red, red) Pareto-dominates (blue, blue) which is the only other possible solution according to RC, i.e., the only other equilibrium, in HiLo.

A rational individual in a situation with strong interdependence will realize that the actors in the situation share a goal which they can collectively achieve. As a consequence, Bacharach claims, the individual will identify with the group. Moreover, if we stick to the common assumption that the rationality of individuals (and the structure of the game) is common knowledge, the fact that individuals identify with the group will also be common knowledge. Although individuals may lack the perfect rationality assumed in theory, similar considerations may well apply to real world individuals. Bacharach, in fact, makes the empirical claim that the perception of strong interdependence stimulates group identification, or more precisely:

The probability of group identification is high if strong interdependence is perceived.

Bacharach argues that reason dictates team reasoning once it is common knowledge that individuals identify with the group. Again, although individuals may lack the perfect rationality assumed in theory, they should be expected to team reason if they identify with the group and perceive others as being alike. This results in another empirical claim, the "reasoning effect" (?, 135 ff.). Group identification stimulates team reasoning, or more precisely:

\section{Common group identification induces a high probability of team reasoning. ( $\quad(\star \star)$}

Strong interdependence is particularly obvious and salient in HiLo and it seems natural to assume that real individuals, just as their ideal rational counterparts, are well aware of the fact that there is a shared goal which they can collectively achieve. In the light of this observation $(\star)$ and $(\star \star)$ yield an empirical hypothesis for the HiLo game: there is a high probability of coordination on the Pareto-optimal outcome in HiLo. In other words: TR (as specified by Bacharach) can explain what is commonly observed but cannot be explained within a pure RC approach.

Sugden agrees: TR can indeed provide a valid explanation for coordination in HiLo games. But he rejects the assumption that common knowledge of group identification is sufficient to identify team reasoning as the unique rational mode of reasoning. According to Sugden, it may still be rational to refrain from team reasoning, even if it is common knowledge that every individual involved also identifies with the group. For Sugden team reasoning just remains one rational mode of reasoning among others. Even if it is common knowledge that all individuals identify with the group, it may remain rational (in the very light of group goals) for a member of the group to follow 
another mode of reasoning, if all others do. So to engage in team reasoning an individual has to be sufficiently assured that others not only identify with the group but also use this mode of reasoning as well. ${ }^{8}$

As a consequence, Sugden demands that any complete explanation by TR has to refer to some form of "assurance". Individuals must have good reason to believe that others identify with the group and employ team reasoning to make their choice. A person that identifies with the group and employs team reasoning as his mode of reasoning is referred to a 'teamer'. For Bacharach, every member of the team is a teamer if group identification (and the rationality of the members) is common knowledge. For Sugden this does not necessarily hold. Individuals must have good reasons to believe that others are teamers, they must have good reasons to believe that the others also have good reasons to believe that group members are teamers, and so on. ${ }^{9}$

But when will this condition be fulfilled? This, again, is an empirical question. Sugden identifies one important source for such assurance: common experience of a shared practice $(?, 135)$. If people regularly observe that others act according to the beneficial scheme and if this is a public experience - everybody observes the regularity, everybody is aware that everybody else does, etc., - then each individual is sufficiently assured that sufficiently many others not only identify with the group but also act on the group's goal in the way TR prescribes.

\section{Instrumental rationality and opportunism}

The founders of TR are firmly rooted within the RC tradition. They were motivated by the shortcomings they found within $\mathrm{RC}$, and so they made an attempt to revise $\mathrm{RC}$ in ways such that many fundamental ideas of RC are preserved while some of its most pressing problems are solved. Moreover, while team reasoning is understood as genuinely rational, it is not claimed to be the only viable form of rational decision-making. As was noted before, TR has a special focus on interaction in which groups of individuals may be identified as units of agency. It is not claimed that new insights to issues outside the range of this focus are actually added.

Thus, TR can be understood as an attempt to advance RC by extending the range of possible subjects of agency and generalizing the fundamental concepts of the theory of individual rationality such that they may be applied to contexts where collectives can be understood as agents. As a consequence, TR is fundamentally related to traditional RC. This becomes particularly lucid by the

\footnotetext{
${ }^{8}$ Compare the problem of equilibrium selection in RC as discussed in section 5.2 above!

${ }^{9}$ This is a rough sketch of the condition only. For a precise formulation see ?, $132 \mathrm{ff}$, and ? for an elucidation of the concept of "reason to believe".
} 
following two observations:

(1) Whatever action TR prescribes to an individual in a certain situation, choosing this action is individually rational in the sense of RC relative to team preferences. Given that the other agents act as teamers the individual maximizes team utility by choosing the act proposed by TR.

(2) RC may be understood as a special case of TR by defining individuals as groups of one. TR then produces the very same theorems as RC on the domain of individuals so defined.

(1) TR is based on a simple insight: a profile of strategies which is proposed by TR maximizes team utility. Hence, there is no other profile with larger team utility and, hence, an individual cannot improve (relative to team utility) by deviating unilaterally. That is, team reasoning is consistent with instrumental rationality in the sense of $\mathrm{RC}$ if individual preferences and team preferences are assumed do be identical. However, TR does more than just adding collective goals to RC. This is manifest in the decision rule (tr). As noted above (tr) serves inter alia to solve equilibrium selection problems which cannot be solved by individual instrumental rationality as defined in RC alone.

(2) is a simple consequence of the guiding idea behind TR. Groups of individuals are introduced as additional units of agency. The traditional assumptions of RC about individuals (i.e., that they are characterized by specific preferences and beliefs) and their ways of decision-making are then transcribed to these new units. In this regard, TR is a genuine amplification of RC: it contains $\mathrm{RC}$ as a special case. Another consequence is that TR mirrors the key assumptions of individual decision-making in $\mathrm{RC}$ in its assumptions about team reasoning.

The essential element of TR's heritage from traditional RC is its rigorous conception of instrumental rationality:

- According to $R C$ all individual goals can be represented by a suitable utility function over the consequences of action, and every motivational force can be represented as a disposition to maximize this utility function in a certain choice situation.

According to $T R$ all team goals can be represented by a suitable team utility function over the consequences of action-profiles, and every collective intention can be represented as a disposition to maximize this utility function in a certain choice situation

- According to $R C$ every individual chooses those options that guarantee (relative to his beliefs) maximum individual utility.

According to TR every team or collective chooses those options that guarantee (relative to common beliefs in the team) maximum team utility. 
- According to $R C$ any individual will choose opportunistically in the following sense: he will instantly detect every opportunity to improve by changing his way to act and use it immediately; he will do so, whenever the opportunity may arise, i.e., in every single situation of choice. Therefore there is no motivational force in considerations like: what will be the overall consequences of me following this route of action in the long run?

According to TR any team will choose opportunistically in the following sense: the team will instantly detect every opportunity to improve by changing the profile of actions by the teamers and it will collectively use it immediately; it will do so, whenever the opportunity may arise, i.e., in every single situation of choice. Therefore there is no motivational force in considerations like: what will be the overall consequences of us following this route of action in the long run?

Opportunism ${ }^{10}$ combines extreme agility and responsiveness to changing circumstances with resolute restrictions on the determinants of choice. On the one hand, opportunistic decision makers unswervingly take any opportunity as soon as it occurs. An opportunistic individual is not governed by customs or habits. his behavior may conform with norms (if this suits his interests), but it is not restricted by norms. he does whatever is best independently of his own past behavior or of what others may think suitable. On the other hand, there is a clear and rigorous restriction on what may influence the decision of an opportunistic decision maker: only the expected future consequences of own individual choices are relevant for opportunistic choice.

Individual opportunism is both, a distinguishing feature of theoretical clarity and analytical rigor in the architecture of RC, and a major source of RC's problems to cope with the empirical regularities of human decision-making. An important example of the problematic aspect of opportunism in RC is the so-called commitment problem as, e.g., illustrated by the Prisoners' Dilemma. Assume player A could commit herself to cooperate if he expected B to cooperate, and B, after being informed on the commitment of A, could commit herself to cooperate. Then, the two could jointly realize the mutually preferred outcome $(\mathrm{C}, \mathrm{C})$. But, although both would benefit from such an arrangement, it is not obtainable to rational individuals in the sense of RC unless they have an external and binding commitment mechanism at their disposal. The sole fact that it is mutually advantageous that both restrict their options does not enable them to actually restrict options, - it cannot be effective as a reason to act. Opportunism is the core reason for this. If the occasion arises each has to choose the optimal option given his beliefs about the actions of the other. As long as a strictly dominant option $\mathrm{D}$ is actually available (as in the PD), opportunism commands to choose it. Opportunism makes

\footnotetext{
${ }^{10} \mathrm{Cf}$. ?, $55 \mathrm{ff}$, for the concept of opportunism as used here.
} 
individuals look solely at the future consequences of their individual choice given their beliefs about other's choices. The fact that regularly following a different scheme of action might be mutually beneficial is irrelevant, as only the choice of each single action is under individual control, but not the choice of a (binding) scheme of action.

HiLo provides another example for the difficulties of opportunistic individual decision-making. That the outcome (red, red) is mutually preferred provides no reason for choosing red because the outcome (red, red) is not an option of an individual. It would provide a reason for an opportunistic individual only if the mutual preferability would somehow justify the expectation that the other would choose red. This would make the option red optimal. But this is exactly what is at issue. It is the very flexibility of opportunistic choices that prevents individual actors from coordination on a mutually advantageous equilibrium. Since opportunistic actors are perfectly responsive to the conditions of choice and absolutely flexible in their reactions they may loose their ground if the crucial determinant of choice is what they expect from others (as is generally the case in strategic interaction).

Individual opportunism is a theoretically elegant conception but it hardly accounts for the complexities of real life human decision-making. Actual human actors are not perfectly free to instantly detect and choose whatever is best. They are subject to the influence of custom and habit, and they are bound by personal and social norms independently of the support of these norms by sanctions. Such observations may, in fact, explain to some extent why the commitment problem as well as problems of coordination are not as pressing for real life individuals as RC would predict.

TR is an attempt to formulate a theory that offers a remedy against the deficiencies of individual opportunism and, at the same time, accounts for real life restrictions and opportunities in decisionmaking processes. According to TR, individuals may restrict the range of their options to those part of an optimal scheme by adopting a team perspective. Thus, they may overcome a commitment problem as represented by the Prisoners' Dilemma game. Moreover, they may cut across the endless regress constituted by the mutual dependence of choice and expectation by introducing the social optimum as an authoritative common point of reference. In both cases the neglect of individual opportunism and its consequences allows for a more realistic account of decision-making.

At least in the form fostered by Sugden TR also gives some account of the influence of custom and habit on decision-making. According to Sugden "assurance" is a necessary condition such that group identification makes individuals act as teamer. The common experience of a shared practice is, as Sugden emphasizes, an important and sufficient condition of such assurance. Hence, according to 
TR, custom plays an important rule in explaining social interaction. Recurring patterns of behavior in a group are taken as evidence for team reasoning to be the prevalent mode of reasoning in the group and - therefore - justify and motivate team reasoning as the appropriate mode of reasoning. Similarly, norms induce commonly shared patterns of behavior and expectations. If these patterns are consistent with the demands of team reasoning they may bring about a shared perception of team reasoning as the appropriate mode of reasoning. This will in turn consolidate the behavioral pattern.

Notice, however, that this account of the influence of custom and social norms on social interaction is limited by the condition that it is team reasoning instead of customs or norms themselves that induces the regularities of conforming behavior. Custom and norms merely explain the stability of team reasoning as a mode of decision-making. But this stability can only be sustained as far as team reasoning continuously prescribes the same route of action. The stability will break down as soon as a change in the environment implies profitable deviations from a team perspective. As noted above, TR assumes the same kind of opportunism for teams as RC does for individuals. Hence, according to TR, teams display the same sort of rigid flexibility and instant responsiveness to changing circumstances as individuals do according to RC. If a new opportunity arises, the team will detect and take it instantly. Understood as units of agency, teams are assumed to be free of habits, custom or normative restrictions. ${ }^{11}$

There is another, more traditional account of behavioral regularities that also sticks to the fundamental assumption of rational decision-making within RC while extending its explanatory power. On this account social norms are defined as specific equilibria ${ }^{12}$ which function as additional constraints on individual decision-making. Thus, norms are understood as the primary source of the regularities and not as a by-product of rational action. Such considerations certainly shed some light on the nature of social interaction in general and on norm guided behavior in particular, but their actual empirical significance is limited. The reason is that - although a general account of the nature of behavioral regularities is given - the theory does not provide an explanation why this or another norm prevails.

\footnotetext{
${ }^{11}$ And so, the commitment problem and the problem of equilibrium selection may reappear on a higher level if different teams interact strategically.

${ }^{12}$ Strictly speaking, only a specific form of norms ("norms of conduct" can be identified with equilibria, see ?? for a detailed account). Well known representatives of the idea that (some) norms are equilibria are, e.g., David Lewis, Michael Taylor, Robert Sugden, Christina Bicchieri and Ken Binmore (see ?, endnote 1 for references). ?? proposes a generalization of the idea based on the theory of epistemic games and the concept of coordinated equilibrium.
} 


\section{Changing accustomed patterns of behavior by team reasoning: introducing uncertainty}

TR not only offers a general account of how people solve coordination problems, it actually generates testable hypotheses on what regularities are to be expected. In the remaining part of this paper we investigate the empirical significance of TR's assumption of opportunistic decision-making on the team level. We focus on a particular form of change in the environment, which offers opportunities to improve on the team goal by changing an accustomed pattern of behavior. This change consists of the introduction of a particular kind of uncertainty regarding the consequences of individual choice.

Our interest in the impact of uncertainty on team reasoning is originally motivated by Bacharach's provoking and in some respect surprising discussion of the demands of TR in case that there is some (known) probability that others will not conform to TR. This sort of problem is well known from rule-utilitarianism: given that, realistically, a certain fraction of the population will act immorally, what rule should the moral person comply with? Donald ? presents "cooperative utilitarianism" as a solution to the problem. This in turn serves Bacharach as a model for the solution of his problem, in his theory of "circumspect team reasoning" (Bacharach, 2006, 130ff; Gold and Sugden, $2007,131)$. The theory displays a radical resoluteness in the team perspective ascribed to individual team reasoners by TR, a resoluteness that, in fact, may well arouse some doubts in the empirical significance of TR.

The theory of circumspect team reasoning is interesting in normative respects but a thorough discussion of the theory would go beyond the scope of this paper. We are interested in the extent of opportunism assumed in TR and the empirical plausibility of these assumptions, which is why we discuss the impact of uncertainty on decision-making in TR. The uncertainty in Bacharach's model of circumspect team reasoning is assumed to be endogenous. However, in order to empirically address the impact of uncertainty we consider a related but exogenously defined uncertainty in a very specific and much simpler setting, namely in variants of the HiLo game. ${ }^{13}$

Our point of departure is a HiLo game with payoffs as given in Figure 5 (with corresponding parameter $\mathrm{a}=1.25$ in the representation as in Figure 3$)$ :

We assume that the given payoffs (the numbers in the bi-matrix) represent standard cardinal utility values, and, as before, we assume that team utilities coincide with individual utilities. Team reasoning then prescribes that every teamer chooses his part in the optimal scheme; accordingly,

\footnotetext{
${ }^{13}$ One major benefit of focussing on exogenously imposed uncertainty is that the latter can be reproduced in the lab, - in contrast to the uncertainty assumed in the theory of circumspect team reasoning. It is hardly possible to exogenously vary the fraction of team reasoners given the same strategic situation in every treatment.
} 


\begin{tabular}{l|ll|l|l|l|}
\multicolumn{1}{c}{} & \multicolumn{1}{c}{ red } & \multicolumn{3}{c}{ blue } \\
\cline { 2 - 6 } red & 5 & 5 & 5 & 0 & 0 \\
blue & 0 & 0 & 4 & 4 \\
\cline { 2 - 6 } & & &
\end{tabular}

Figure 5: Basic HiLo game

teamers will coordinate on the Pareto-optimal outcome (red, red).

We will now, consecutively, introduce two slight modifications of the game. First, we consider the game resulting from the one above, if a random mechanism partly determines the choice of one of the two players, in the following referred to as person A. Depending on the outcome of this mechanism A can either freely choose between red and blue, with probability $\omega$, or is forced to choose blue with probability $1-\omega$. The other player, person $\mathrm{B}$, is not informed on the outcome of this random mechanism. However, the existence of such a mechanism and $\omega$ are common knowledge.

Payoffs are again symmetric across players. Figure 6 represents the expected outcomes (in terms of team utilities) for the different combinations of strategies. As long as $5 \omega>4-4 \omega$, i.e., $\omega>\frac{4}{9}$, the game is still a coordination game with equilibria (red, red) and (blue, blue). Therefore, both choices red and blue are rationalizable from an $\mathrm{RC}$ point of view. If an individual has reason to expect his partner to choose red, choosing red is his rational response, and a corresponding statement applies to expecting the partner to choose blue: it is a sufficient reason to choose blue oneself.

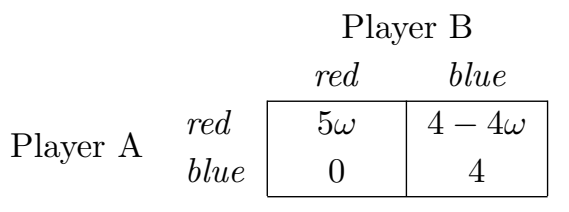

Figure 6: One-sided uncertainty

From a team perspective (red, red) is the unique optimal outcome as long as $5 \omega>4$. So TR predicts that teamers will choose red in the role of person A as well as in the role of person B if $\omega>0.8$, and blue if $\omega<0.8$. As TR determines a unique best scheme of action as long as $\omega \neq 0.8$, choices of teamers are uniquely defined.

We conclude: if the likelihood that person A is forced to choose blue is high enough (larger than 0.2 ), then teamers will take the opportunity to maximize expected (team) earnings by changing to blue.

The experience of a shared practice in the standard HiLo may assure players that team reasoning is the prevailing mode of reasoning among individuals who identify with the group. But a shared practice owns no independent momentum of inertia, as one might expect from experience with common social practices. TR predicts that (red, red) is abundant as soon as (blue, blue) becomes 
optimal from the team's perspective. Yet, (red, red) could still serve as a convenient focal point for coordination.

In a second step, we extend this kind of uncertainty to both players. Consider the modified HiLo game in which both individuals are independently confronted with a chance move that might force them to choose blue. Two formally identical random mechanisms determine - independently - for each of the individuals whether he can freely make his choice (with probability $\omega$ ) or is forced to choose blue (with probability $1-\omega$ ). While outcomes of the mechanisms are only communicated to the respective individual but not to his partner, the mechanism as such and the value of $\omega$ are again common knowledge. Payoffs remain symmetric. Figure 7 represents the expected outcomes for the different combinations of strategies.

This case of two-sided uncertainty is formally identical to the uncertainty that gave rise to Bacharach's introduction of circumspect team reasoning. ${ }^{14}$ Hence, the analysis to follow perfectly accords with Bacharach's corresponding discussion (see in particular ?, 132 f.).

The game in Figure 7 is still a coordination game with equilibria (red, red) and (blue, blue) as long as $5 \omega^{2}+4(1-\omega)^{2}>4-4 \omega$, i.e., as long as $\omega>\frac{4}{9}$. (red, red) is the unique optimal outcome as long $5 \omega^{2}+4(1-\omega)^{2}>4$. Hence, TR prescribes to choose red if $\omega>\frac{8}{9}$ and blue if $\omega<\frac{8}{9}$. As with the first modification above: if the likelihood that a partner is committed to choose blue is high enough, then team reasoners will take the opportunity to maximize expected (team) earnings by changing to blue.

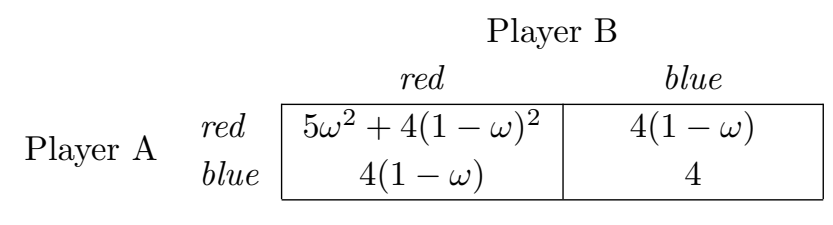

Figure 7: Two-sided uncertainty

Hence, if $\frac{4}{5}<\omega<\frac{8}{9}$ team reasoners will coordinate on (blue, blue) in case of two-sided uncertainty, while they will coordinate on (red, red) in the corresponding case of one-sided uncertainty.

This result may come as a surprise: at first sight the situation of an actor under two-sided uncertainty after being informed that he may freely choose seems to resemble the situation of actor

\footnotetext{
${ }^{14}$ There is a slight, but relevant substantial difference, though: while there is a known proportion $1-\omega$ of nonteamers in the case of circumspect team reasoning, all individuals in case of two-sided uncertainty as discussed here are assumed to be teamers. In the circumspect team reasoning scenario the non-teamers deliberately choose a default strategy s* (corresponding to blue in our case) while in case of two-sided uncertainty teamers are forced to choose blue. This difference may be of some relevance in relation to the empirical inclination of individuals to identify with the group. If I know that there is a considerable number of people who do not identify with the group then I might myself be less motivated to identify with the group. So group identification seems to be more precarious in the circumspect team reasoning scenario.
} 
$\mathrm{B}$ under one-sided uncertainty in all relevant respects.

Assume $\frac{4}{5}<\omega<\frac{8}{9}$. Consider the situation of an actor A after being informed that he is free to choose. Given this private information the expected utility of the profile (red, red) is $5 \omega$. So (red, red) is the optimal profile for the team from A's perspective, - it would be best if both choose red. Of course, the partner $\mathrm{B}$ does not have the same information. $\mathrm{B}$ does not know that $\mathrm{A}$ is free to choose; therefore it may seem questionable to A that B will do his part in the optimal profile. But notice that, if $\mathrm{B}$ actually gets the chance to choose, his situation will be exactly like the one we have just discussed. So B will also find that (red, red) is the optimal profile. Finally, from the perspective of any individual free to choose (red, red) is the optimal profile; and rational individuals, whether teamers or not, will know this. However, teamers should neglect their private information about their own situation in their decision-making, which is why - seemingly contrary to their interest TR defines (blue, blue) as the optimal profile in case of $\frac{4}{5}<\omega<\frac{8}{9}$. Is it possible, that from the perspective of every player who gets the opportunity to choose (red, red) is the optimal scheme of action in terms of group goals, while (blue, blue) is in fact optimal?

It is! Note that the argument above considers only the expected gains of those that are free to choose. The expected loss of those that are forced to choose blue are not taken into account. But in case of $\frac{4}{5}<\omega<\frac{8}{9}$ these losses outweigh the potential gains of those left free to choose. Individual opportunism is at the heart of the strategic calculus unfolded above. It is true: for those that actually can control their choice (red, red) is the optimal profile. So if they mutually control the outcome - as TR assumes for the team as a whole - they should choose red. Moreover, if all choose accordingly each does his part in the profile that is optimal from his own perspective, - some because they already know that they actually control their choice, others because their choice is irrelevant anyway. But, as in the classical PD: if everybody chooses the best means available to achieve his aims (which, in our case, are actually shared aims) this does not necessarily result in an optimal outcome for each. In fact, the expected outcome for everyone is suboptimal.

TR offers a cure for the detrimental narrow-mindedness of individual opportunism among those that by coincidence acquire the power to decide. Team reasoning yields better results for the team and the individuals on average, by disregarding the individual and concentrating on the collective perspective only. The argument above considers for each actor in a team the case that he is free to choose while it is unknown whether the partner is also free or not. But these cases overlap and they are not exhaustive. To determine the optimal profile for the team one must consider the following four cases which are pairwise disjoint and mutually exhaustive instead: 
(i) first player free, second bound to choose blue;

(ii) first player bound, second free;

(iii) both players free; and

(iv) both players bound.

Taking all these cases and their respective probabilities into account we get the result stated above: team reasoners will choose red if $5 \omega^{2}+4(1-\omega)^{2}>4$, and blue if $5 \omega^{2}+4(1-\omega)^{2}<4$. Team reasoning can, thus, overcome suboptimal choices suggested by individual opportunism to those that effectively control their choices.

However, opportunism remains a crucial characteristic of decision-making according to TR, albeit opportunism concerning team actions in the light of team goals. If the environment changes from $\omega>\frac{8}{9}$ to $\omega<\frac{8}{9}$ teamers will, again, instantly and concurrently change from coordinating on red to coordinating on blue. A shared practice is entirely volatile under the regime of team reasoning. TR predicts that it will immediately change if the probability of a forced move exceeds $\left.\frac{1}{9}\right)$.

Finally, note the problem of two-sided uncertainty as discussed above may also be framed as a problem of using private information. Individual opportunism demands that every player should use all the information available to his in making his decision. This is largely what drives the argument suggesting that rational players should act in exactly the same way in cases of one-sided and two-sided uncertainty if the parameter $\omega$ is identical. But team reasoning demands to refrain from using information not mutually available to all individuals in the team alike. The simple reason is that every teamer must be in the position to determine the team solution to contribute his part, so the solution cannot depend on information that is not available to all. While TR's tendency to transgress individual opportunism may appear as a step towards a more realistic account of social interaction in general, neglecting private information may well seem a too demanding requirement for real human individuals.

\section{Experimental evidence}

\subsection{Experimental design}

We conducted a laboratory experiment to test behavior differences in the situations discussed in the preceding section. The experiment involved three different treatments in a between-subjects design, 
and each treatment consisting of three parts. The exogenous variation across treatments referred only to the second part of the experiment.

In the beginning of the experiment, every subject was paired with another participant, in the following referred to as his 'team member' or 'partner'. These 'teams' remained the same for the rest of the experiment. In every team one subject was randomly assigned the role of person A, the other subject the role of person B; roles were in fact only effective in Part 2 as described below. Although team members were anonymous throughout, we encouraged team identity by framing pairs as 'teams' in the instructions. ${ }^{15}$

In Part 1, each team played a series of five HiLo games with payoffs (in Euro) as given in Figure 5. Feedback about the other team member's choice and the payoff associated with the team's strategy profile was given after every round. ${ }^{16}$ By being engaged in a sequence of interactions subjects were induced to identify with their team and to coordinate on one equilibrium outcome. Moreover, by controlling whether team members were able to perfectly coordinate throughout in Part 1, we may assume that group identification as required in TR was satisfied. First, subjects gained experience in the series of HiLos, which we consider sufficient as 'assurance' in the sense of Sugden. Second, the HiLo game displays 'strong interdependence' as is required according to Bacharach. ${ }^{17}$

In Part 2, participants played a one-shot modified HiLo game with uncertainty as defined in either Figure 6 or Figure 7, depending on the treatment. Treatments differ with respect to the kind of uncertainty and the probability $\omega$. We implemented one-sided uncertainty with $1-\omega=\frac{1}{6}$ (treatment ONE-L(ow risk)) and with $1-\omega=\frac{1}{3}$ (treatment ONE-H(igh risk)), and two-sided uncertainty, again with $1-\omega=\frac{1}{6}$ (treatment TWO-L(ow risk)). Again, the team member's choice and associated payoff was communicated ex-post.

In the ONE-sided treatments, only one team member, namely person $\mathrm{A}$, faced a chance move which forces his to choose blue with probability $1-\omega=\frac{1}{6}$ in ONE-L, and with probability $1-\omega=\frac{1}{3}$ in ONE-H. Importantly, person B was not informed on the realization of this chance move at any point in the experiment. In the TWO-sided treatment, both team members faced the risk of being compelled to choose blue, with probability $1-\omega=\frac{1}{6}$ and independently of each other. Again, subjects were not informed about the realized outcome of their partner's chance move. ${ }^{18}$ As in

\footnotetext{
${ }^{15}$ The instructions for treatment ONE-L (described below) are provided in appendix A; the instructions for other treatments are available from the authors.

${ }^{16}$ To avoid any income effects, if this part was selected for payoff, only one round was randomly determined to be paid out to subjects. More details on the experimental procedures are provided in section 5.6.2.

${ }^{17}$ Subjects that proved unable to coordinate in Part 1 were excluded from the data set.

${ }^{18}$ To describe the random mechanism in an intuitive way to subjects the realization of chance moves was implemented as follows. In the ONE treatments Person A was assigned an identification number, i.e., an integer ranging from 1 to 6 in ONE-L and ranging from 1 to 3 in ONE-H. Accordingly, in the TWQ treatment Person A and Person B were
} 
Part 1 the condition of strong interdependence is satisfied in all treatments, and we assume that assurance gained from the series in Part 1 is still effective.

In Part 3, we elicited individual risk attitudes using choice lists with 16 different decision items (see, e.g., ?). In each of these items participants had to choose between receiving a certain amount of money and participating in a lottery. While the lottery was kept constant across all decisions, the certain amount increased continuously from the first to the last decision item. Finally, at the end of the experiment participants completed a questionnaire on socioeconomic characteristics.

\subsection{Experimental procedures}

Experimental sessions were conducted between June and October 2013 at the Frankfurt School Laboratory, Frankfurt School of Finance and Management, Germany. Participants were recruited from the pool of resident students at Frankfurt School. In total 103 subjects participated in our study. ${ }^{19} 23.3 \%$ were female, the average age was 22 years, and all of them had a business or management background in their studies. Detailed instructions were handed out to participants in the beginning of every part, i.e., not before the preceding part was finished. Instructions were read out aloud by the experimenter in front of participants to exclude the opportunity that participants received private information. Everyone was given enough time to carefully look at them and ask questions before and during the experiment, which were answered in private.

In Part 1, subjects necessarily received feedback about their team members choice and, hence, about the monetary outcome realized by their mutual actions. To avoid any income effects within Part 1 and across Part 1 and 2, for each team, either Part 1 or Part 2 was randomly and equally likely selected for payment. Further, if Part 1 was selected, only one round was again randomly drawn to be payoff-relevant. Additionally, subjects received their earnings from their individual decisions in Part 3. In this part only one of the 16 decision problems was again randomly selected for payoff. ${ }^{20}$ If a subject chose lottery A for this particular problem, the computer performed a random draw given the respective distribution and independent from other subjects, to determine the lottery outcome. On top, participants received a show-up fee of $€ 4.00$. On average participants earned $€ 13.00$ (approximately $\$ 17.50$ at the time of the experiment). All of what is written above was common knowledge.

each assigned an identification number between 1 and 6. Then, a virtual dice (6-sided or 3-sided, respectively) was rolled by the computer. In case the dice coincided with the subject's ID, his choice was set to blue, while he was free to choose in the other case.

${ }^{19}$ As the experiment necessitates an even number of subjects, in one session a member of the student assistants staff participated; his data are not included in the analysis.

${ }^{20}$ All random draws were implemented by the computer. 


\subsection{Hypotheses}

The condition of strong interdependence is satisfied in every game of Part 1 and Part 2 in the experiment. We further assume that successful coordination on (red, red) in Part 1 generates 'assurance' in the sense of Sugden: subjects will have common reason to believe that their partner identifies with their team and makes his decision accordingly. Thus, for those who manage to coordinate in Part 1 TR may predict subjects' decisions in Part 2. Hence, we restrict our analysis to those individuals that continuously succeeded in coordinating on the profile (red, red) in Part 1 and assume strong interdependence and assurance to be satisfied in what follows.

If both partners are teamers, then TR predicts coordination on (red, red) in ONE-L and coordination on (blue, blue) in ONE-H and TWO-L. If team reasoning is in fact the prevalent mode of reasoning in coordination problems, then this results in the following hypotheses for behavior within each treatment in Part $2 .^{21}$

Hypothesis 1 (Strategy profiles).

(H01) A considerable majority of the subjects chooses red in treatment ONE-L.

(H02) A considerable majority of the subjects chooses blue in treatment ONE-H.

(H03) A considerable majority of the subjects chooses blue in treatment TWO-L.

Participants were randomly assigned to treatments, and thus the fraction of teamers and nonteamers may be assumed to be similar across treatments. This yields the following hypotheses for differences across treatments in Part 2:

Hypothesis 2 (Treatment differences).

(H11) The fraction of subjects who choose red in treatment ONE-L is significantly higher compared to treatment $O N E-H$.

(H12) The fraction of subjects who choose red in treatment ONE-L is significantly higher compared to treatment $T W O-L$.

(H13) The fraction of subjects who choose red in treatment TWO-L is not significantly different from the fraction of subjects who choose red in treatment ONE-H.

\footnotetext{
${ }^{21}$ In our theoretical discussion of the HiLo game (with and without uncertainty) we assumed expected utility theory with near-linear utility functions. Using the data from Part 3 we controlled for this conditions as outlined in section 6.5 .
} 


\subsection{Results}

We first comment on behavior in Part 1 before we analyze behavior in Part 2. Results from Part 3 , in which we controlled for any effects of risk attitudes, are addressed within the discussion in section 6.5.

\subsubsection{Part 1}

Although RC does not imply a prediction for this series, it is not surprising that all but one team consistently coordinated on the strategy profile (red, red). For the reasons explained above, the data of those two subjects who failed to coordinate is excluded from the analysis of Part 2. This leaves us with 101 observations, 36 observations in treatment ONE-L (18 subjects in the role of person A, 18 in the role of person B), 45 observations in treatment ONE-H (23 in role A, 22 in role B), and 20 observations in TWO-L (role A and role B were perfectly congruent).

\subsubsection{Part 2}

In ONE-L, two A players were forced to choose blue in Part 2; in ONE-H, eight A players were forced to choose blue; in TWO-1/3, two subjects were forced to choose blue. All numbers provided in the following analysis refer only to those subjects that could actually make a choice, i.e., we do not count enforced choices.

\section{Strategy profiles}

TR predicts that in ONE-L teamers should choose red, and we in fact observe that a significant majority chooses accordingly (two-sided-binomial test; p-values 0.001, 0.035, 0.013, for the pooled sample, for A and B players, respectively). ${ }^{22}$ Figure 8 displays the fraction of subjects who chose red for each treatment. While more than $81 \%$ chose red in ONE-L, this fraction shrinks to $67 \%$ in TWO-L, and to $42 \%$ in ONE-H. This clearly supports Hypothesis (H01). Consistent with TR, we also observe a lower frequency of red choices in both other treatments. While in ONE-H a majority of subjects chose blue, in support of (H02), a majority of subjects still chose red in TWO-L, contrary to (H03). However, neither (H02) is sufficiently supported nor can (H03) be rejected by the data: we cannot reject the hypothesis that the fraction of red choices is statistically significantly different

\footnotetext{
${ }^{22}$ The statistical analysis is conducted in the most conservative way, treating observations of team members not as independent observations, given that team members interacted in Part 1. However, given that we drop those observations where team members failed to coordinate throughout in Part 1, and given that participants and, in particular, team members stayed anonymous during the experiment, we may have also treated team observations as independent, in which case our results remain unchanged.
} 
to $50 \%$ (two-sided binomial tests; p-values $0.238,0.508,0.508$ in TWO-L, and 0.473, 1.0, 0.238 in ONE-H, again for the pooled sample, for A and B players, respectively).

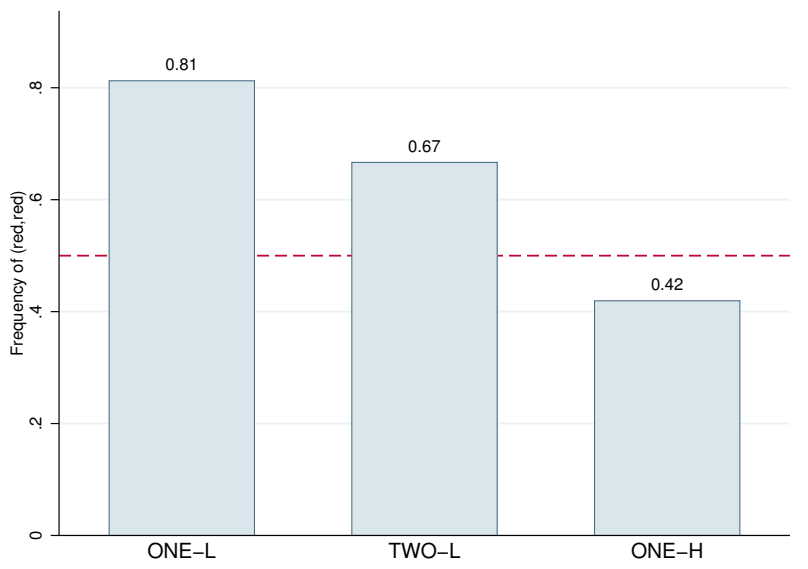

Notes: numbers only refer to choices of subjects who were eligible to make a choice, i.e., enforced decisions of A players in ONE-H and ONE-L, and of A or B players in TWO-L are excluded.

Figure 8: Frequency of red choices by treatment

In TWO-L, we can at least reject the hypothesis that the average fraction of red choices in TWO-L is smaller than 0.48 (p-value 0.088, one-sided binomial test). This yields evidence against the prediction that a considerable majority generally chooses blue, i.e., against (H03). Overall, given the limited number of observations, we find some evidence against (H03) in TWO-L, and some evidence for (H02) in ONE-H.

\section{Strategy profiles by role}

According to TR team members should coordinate on the optimal strategy profile which is symmetric in roles. That is, predictions (H01), (H02) and (H03) do not depend on whether players are assigned role $\mathrm{A}$ or $\mathrm{B}$, i.e., face the uncertainty of being forced to choose blue themselves. Hence, in a next step, we differentiate by role. Table 1 provides the fraction of subjects who chose red, by role and treatment.

\begin{tabular}{lcccc}
\hline Treatment & Player B & Player A & Player A \& B & $N$ \\
\hline ONE-L & $82.35 \%$ & $80.00 \%$ & $81.25 \%$ & 34 \\
TWO-L & $66.67 \%$ & $66.67 \%$ & $66.67 \%$ & 20 \\
ONE-H & $33.33 \%$ & $53.85 \%$ & $41.94 \%$ & 37 \\
\hline \hline
\end{tabular}

Notes: enforced decisions again are excluded.

Table 1: Fractions of red choices by treatment and role

Both, with low risk of blue enforcement in ONE-L and TWO-L, role does not significantly matter. 
In contrast, we observe a striking difference between choices of A players and B players in ONE-H. The overall effect in this treatment - i.e., that a majority chose blue, which is in line with (H02) - is apparently driven by the behavior of B players. While $67 \%$ of them chose blue only $46 \%$ of $\mathrm{A}$ players chose blue, contrary to what TR predicts. ${ }^{23}$ For B players we can again reject the hypothesis that the fraction of subjects who chose blue is smaller than $52 \%$ (p-value 0.088, one-sided binomial test), providing mild support for (H02) and B players. However, there is considerable doubt that (H02) also applies to role A players.

Result 1. We find significant evidence for (H01); we do not find significant evidence for (H03) and only mild support for (H02). We find evidence for (H02) only for B players and not for A players, which is not consistent with TR.

\section{Treatment differences}

According to TR the distribution of choices should also differ between treatments. First, we find significant evidence for (H11): the frequency of red choices is significantly different between ONE-H and ONE-L ( $\chi^{2}$ - and Fisher exact test, p-values 0.006 and 0.005 , respectively, collapsing over teams). However, if we distinguish between player A and player B (see Figure 9) we again find that this difference is significant only for B players ( $\mathrm{p}$-values 0.003 for B, 0.139 for A). This finding is not consistent with TR, since theory does not predict different effects for different roles.

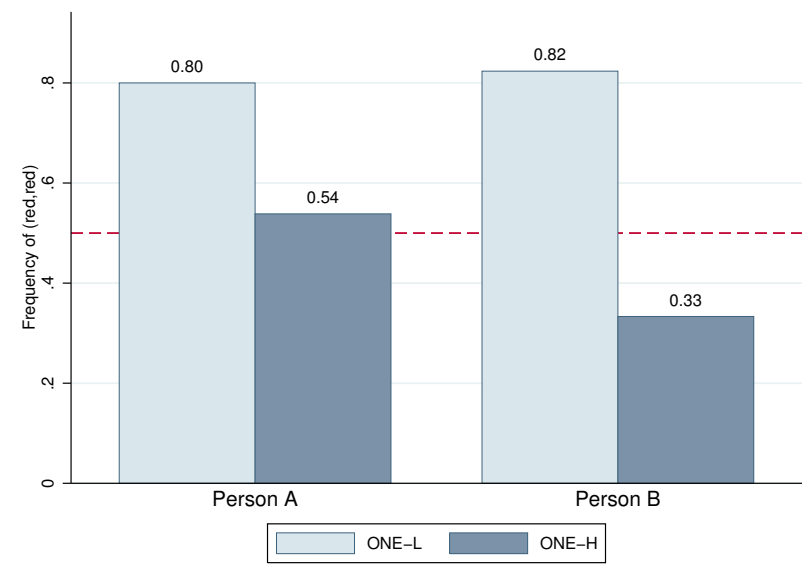

Notes: enforced decisions again are excluded.

Figure 9: Treatment effects for one-sided uncertainty by role

Second, TR would predict a significant drop of red choices from ONE-L to TWO-L (H12). We

\footnotetext{
${ }^{23}$ Using a $\chi^{2}$-test the difference between the fraction of red choices is not significantly different between players $\mathrm{A}$ and B, p-value 0.253 . Yet, our sample only counts $13 \mathrm{~A}$ players and $18 \mathrm{~B}$ players.
} 
do, in fact, observe a moderate drop of red choices, but this change is not significant at a satisfying level (p-values 0.351 and 0.315, respectively, collapsing over teams).

Lastly, our observations are neither consistent with (H13). While theory does not predict a difference between ONE-H and TWO-L (H13), we only find that the frequency of red choices is marginally significantly lower in ONE-H compared to TWO-L (p-values 0.130 and 0.131, respectively, collapsing over teams). But again this result is driven by role B behavior (p-value 0.010). The behavior of A players is not significantly different in the two treatments (p-value 0.548). Although the latter might be consistent with theory, the fact that the majority of A players choose red, as noted above, is not. Overall, the observed treatment differences again do not support TR's predictions:

Result 2. We find significant evidence for (H11); we do not find significant evidence for (H12) and (H13). Further, (H11) is only supported by behavior of B players, but not of A players.

\section{Coordination Rates}

We briefly comment on coordination rates and realized profits. Surprisingly, in both treatments with one-sided uncertainty, the fraction of teams who managed to coordinate on either (red, red) or (blue, blue) is quite high - and similar. While 64.7\% coordinate in ONE-H, even $67.6 \%$ coordinate in ONE-H where the probability that player A is forced to choose blue is even higher. Under twosided uncertainty, only $40 \%$ succeed to coordinate. However, in ONE-H, of those who manage to coordinate, only $28.0 \%$ ultimately coordinate on (red, red), but $72 \%$ on (blue, blue). In contrast, $90.9 \%$ conditionally coordinate on (red, red) in ONE-L, and at least $75 \%$ in TWO-L, under two-sided uncertainty. Under one-sided uncertainty, $\chi^{2}$-tests show that successes in conditionally coordinating on (red, red) are significantly less frequent under high compared to low uncertainty (p-value 0.001, collapsing over teams). It is also significantly smaller in ONE-H compared to two-sided uncertainty (p-value 0.074). Put differently, conditionally coordinating on (blue, blue) is significantly more frequent in ONE-H compared to both other treatments. Finally, these coordination rates naturally translate into average earnings which are highest in ONE-L and lowest in TWO-L.

\subsection{Discussion}

Result 1 and Result 2 reveal that we do not find good evidence for opportunistic decision-making according to TR. Consistent with TR's prediction (H01) we observe that a significant majority of subjects choose red under the condition that only player A faces the uncertainty of being forced to choose blue and that the probability of blue enforcement is rather low (1/6). Also consistent 
with TR's prediction (H02) we observe that a majority of subjects choose blue under one-sided uncertainty if the probability of blue enforcement is rather high $(1 / 3)$.

However, if we discriminate for roles A players and B players appear to behave differently in the asymmetric situation of one-sided uncertainty with a high risk of player B being forced to choose blue. In ONE-H the majority of A players still choose red instead of blue, i.e., not consistently with TR's prediction. This result makes us doubt whether the initial evidence for (H01) actually supports TR. The behavior under two-sided uncertainty provides further arguments against TR. The majority of subjects chose red in TWO-L, contrary to the prediction (H03). One possible argument might be that a subject who learned that he is free to make a choice, might perceive the risk that the other is forced to choose blue to be rather low (1/6). In this way, he would indeed make use of his private information which is not available to his group member.

Treatment comparisons also yield mixed results with respect to TR's predictions. While (H11) seems to be supported at a first glance, distinguishing between roles again shows that (H11) is supported for B players only. Finally, we observe a significant difference between ONE-H and TWO-L and, thus have to reject (H13), which in turn contradicts TR. Apart from the weak support for TR when averaging over both roles, the differences in behavior and treatment effects across roles is generally inconsistent with TR.

\subsubsection{Alternative theories of choice and the importance of private information}

The only solid support in favor of TR seems to be the significant evidence for (H01). However, this result is also consistent with other theories of choice. In particular, a simple variant of cognitive hierarchy theory (?) with custom as an input delivers the same prediction, assuming sufficient similarity between choice situations in Part 1 and Part 2:

(0) Assume that level 0 individuals tend to stick to an accustomed pattern of behavior if choice situations are sufficiently similar. In our set up, after successful coordination in Part 1, level 0 individuals should choose red in Part 2.

The same remains true if strategic reasoning is added:

(1) Assume that level 1 individuals believe that others act in line with (0) and maximize utility given this belief. If the fraction of level 0 players that choose red is sufficiently high, it is rational for level 1 players to choose red as well.

(2) Assume that level 2 individuals believe that a certain fraction of the others act as level 0 
players while others act in line with (1). And assume, again, that level 2 players maximize utility given this belief. In this case it is, again, rational for them to choose red.

Obviously, for every higher level of strategic reasoning we get the very same result. So the assumption that our population is composed of subjects using different levels of strategic reasoning in the sense of cognitive hierarchy theory combined with the assumption that custom produces predominantly red choices among level 0 players yields hypothesis (H01): a considerable majority will choose red. What we learn from these considerations is: the alleged support for TR by the confirmation of (H01) in treatment ONE-L is quite weak. TR does not explain our observations better than the assumption that behavior is simply determined by habit or custom or a theory that combines such an assumption with some strategic reasoning of any level as in cognitive hierarchy theory.

Moreover, this simple cognitive hierarchy model is not only on a par with TR in explaining our observations under one-sided uncertainty, in contrast to TR it also offers an explanation of our observation that a majority of the subjects chose red under two-sided uncertainty:

(0) Assume that level 0 individuals tend to stick to an accustomed pattern of behavior if choice situations are sufficiently similar. In our case, after successful coordination in Part 1, a majority of level 0 individuals choose red in Part 2.

Again, this remains true under strategic reasoning:

(1) Assume that level 1 individuals believe that others act in line with (0) and maximize utility given this belief. Given our assumptions for level 0 it is rational for them to choose red, if the probability p that level 0 players choose red and the probability $\omega$ that they are free to choose are sufficiently high.

Obviously the same applies to higher level strategic reasoning. Therefore cognitive hierarchy with an input of (sufficiently effective) custom on level 0 produces a prediction consistent with our observation.

A particularly striking observation is the asymmetry between role A and role B behavior in ONE-H. Remember that the game subjects are playing in ONE-H is given by a symmetric strategic form with symmetric payoff outcomes - although only one team member faces the risk of not being allowed to choose. Whatever strategy is optimal for A must also be optimal for B. If there is a significant difference between the choices of role A and role B players this must be due to an asymmetry in the interaction that is not captured in the strategic form. Such an asymmetry arises in the course of the game when player A obtains private information on whether he can make his 
choice or not. In TR this asymmetry is not taken into account. In the simple cognitive hierarchy model it is. In fact, because cognitive hierarchy accounts for this asymmetry it also provides an explanation of the asymmetry in behavior that we observe in ONE-H:

(0) Assume that level 0 individuals tend to stick to an accustomed pattern of behavior if choice situations are sufficiently similar. In our case, after successful coordination in part 1 , level 0 individuals choose red with some probability p significantly larger than $1 / 2$.

Assuming sufficient similarity between choice situations in Part 1 and Part 2 of ONE-H custom would produce a majority of red choices. The asymmetry gets in, when strategic reasoning is considered in higher levels:

(1) Assume that level 1 individuals believe that others act in line with (0). Consider a level 1 player in role A maximizing utility given this belief. If $p>\frac{4}{9}$ his optimal choice is red. In contrast a level $1 \mathrm{~B}$ player faces a blue choice of his partner with probability $1-p \cdot \frac{2}{3}$. If $p<\frac{2}{3}$ his optimal choice is blue. Thus, for $\frac{4}{9}<p<\frac{2}{3}$, we get: level 1 A players will choose red, level 2 B players will choose blue.

This pattern is reproduced for higher levels. A players of level $n$ will choose red as long as they believe that the overall fraction of $\mathrm{B}$ players of lower levels who choose red is larger than $\frac{4}{9}$. B players will choose blue as long as they believe that the overall fraction of A players choosing red is smaller than $\frac{2}{3}$.

If the population is suitably composed of actors of different reasoning levels (e.g. composed of all levels with the fraction of level 0 players being sufficiently high and the fraction of level 1 players being comparably low) this will create a pattern as the one we observe: a majority of A players choose red, while a majority of the B players choose blue.

We are not claiming that cognitive hierarchy theory offers the correct and unique explanation for the behavior in our experiment. However, considering cognitive hierarchy theory as a theory of choice sheds some light on why TR seems to fail as an explanation. In particular, our discussion highlights the two main weaknesses of TR as an empirical theory of choice:

First, bygones are bygones. As a theory of opportunistic choice TR cannot account for the influence of past behavior on future choice. A regularity observed in the past can inform individuals on the type of players they are paired with - in Sugden's version of TR it may inform us on team identification. But it has no direct impact on the behavior to be expected. Therefore TR cannot account for habitual and customary behavior and its indubitable impact on social interaction. 
Second, TR confines the individual decision maker to a team perspective that can be shared by everyone. This severely restricts the use of arguments based on private information gained in the course of the game to determine optimal choice. In the experiment A players appear to use their private information on their realized outcome, although TR prohibits them to do so.

\subsubsection{Risk attitudes}

So far, in theory as well as empirically, we neglected the potential influence of risk attitudes, we simply assumed near-linear utility functions. Risk aversion which is predominantly found among subjects in experiments does not have a significant effect on the main predictions of TR. We still elaborate on possible confounds at this point.

From a TR perspective, in Part 2 of the experiment subjects choose among two lotteries given by the payoffs of the two equilibrium outcomes. ${ }^{24}$ In all treatments group members gain 4 currency units if both coordinate on (blue, blue).

In ONE-L by coordinating on (red, red), subjects enter a lottery that yields 0 with probability $1-\omega=\frac{1}{6}$, and 5 otherwise. Risk neutral and risk seeking team reasoners as well as moderately risk averse individuals are predicted to choose red (as assumed in (H01)). In contrast, if individuals are sufficiently risk averse, they should rather go for blue. Thus, taking risk aversion into account might result in a slight variation of Hypothesis (H01). This was in fact the only hypothesis that is actually supported by our data with satisfying significance.

In ONE-H, coordinating on (red, red) implies the lottery that yields 0 with probability $1-\omega=\frac{1}{3}$ and 5 otherwise. In TWO-L by coordinating on (red, red) individuals enter a lottery that pays 4 with probability $(1-\omega)^{2}=\frac{1}{36}, 5$ with probability $\omega^{2}=\frac{25}{36}$, and 0 otherwise. In both treatments (since expected values are smaller than 4) risk neutral and risk averse team reasoners as well as moderately risk seeking individuals are predicted to choose blue (as assumed in (H02) and (H03)). Only sufficiently risk seeking individuals should go for red. If subjects are predominantly risk neutral or risk averse (H02) remains unaffected by taking risk attitudes into account.

Hence, in ONE-L sufficiently risk averse teamers might choose blue, while in ONE-H and TWO-L sufficiently risk seeking teamers might choose red. Assuming that risk aversion predominates risk seeking we get a slight weakening of (H11) and (H12): some risk averse individuals are predicted to choose blue in ONE-L as they do in ONE-H and TWO-L, thus weakening the difference between treatments ONE-L and ONE-H and TWO-L, respectively.

\footnotetext{
${ }^{24}$ To make the following argument we assume that individuals simply transcribe their individual utility function to the team, and thus, under expected utility theory, transfer their individual risk attitude onto the group.
} 
Comparing ONE-H and TWO-L as in (H13) and assuming that risk aversion predominates risk seeking we get only a very a slight weakening of $(\mathrm{H} 23)$ because team reasoners will only act differently in the two treatments if they are risk seeking in a quite peculiar way. They must evaluate ' 4 for sure' better than the lottery that delivers 0 with probability $1-\omega=\frac{1}{3}$ and 5 otherwise, but worse as the lottery that delivers 4 with probability $(1-\omega)^{2}=\frac{1}{36}, 5$ with probability $\omega^{2}=\frac{25}{36}$ and 0 else. Such an individual will - according to TR - choose red in TWO-L but blue in ONE-H. So, to the extent that such individuals are among the subjects, TR predicts a weakening of (H 23). These subjects will behave differently in the two treatments.

The sample size of our experiment is quite limited in order to identify any of the above effects. Nevertheless, we collected data about subject's risk attitudes to test whether any behavioral changes that might support or undermine TR are due to risk rather than to team reasoning. In Part 3, we included a choice list to elicit risk attitudes w.r.t. a risky lottery related to the strategic (risky) situation in the experiment. In 16 decisions subjects had to choose between certain amounts of Euros $(0.5 ; 1 ; 1.5 ; 2 ; 2.5 ; 3 ; 3.2 ; 3.4 ; 3.6 ; 3.8 ; 4 ; 4.2 ; 4.4 ; 4.6 ; 4.8 ; 5)$ and a risky lottery that pays $€ 0.00$ with probability $1 / 6$, and $€ 5.00$ with probability $5 / 6$. In ONE-H subjects completed an additional list in which the lottery pays $€ 0.00$ with probability $1 / 3$, and $€ 5.00$ with probability $2 / 3$. The switching point at which a subject switches from choosing the lottery to choosing the certain amount defines the individual's certainty equivalent (CE), i.e., the smallest amount preferred to the lottery. ${ }^{25}$ We define subjects as risk averse if their certainty equivalent is smaller than the expected value of the respective lottery.

We find that at least $80 \%$ of subjects are risk averse $(80.65 \%$ in ONE-H, $84.4 \%$ in ONE-L and 94.5\% in TWO-L), in line with the assumption made above. However, we cannot identify any of the relationships between risk aversion/seeking and the likelihood to choose red, which we considered. Risk averse and risk seeking individuals are nearly equally likely to choose red, see Table 2 in appendix B for details on choices by treatment, role and risk attitudes.

\section{Conclusion}

We do not find good evidence for opportunistic team reasoning as a guide to coordination in our experiments. Our observations suggest that individuals tend to stick to behavioral patterns they are in some way or other accustomed to. As a theory of opportunistic choice TR categorically contradicts such influence of past behavior on future choice. Moreover, we find significant differences

\footnotetext{
${ }^{25}$ Additionally, in the questionnaire conducted at the very end of the experiment we asked subjects to assess their risk attitude on a scale from 1 to 10, however, this was not incentivized.
} 
in the behavior of subjects in accordance with their individual and particular situation of choice. Individuals may obviously take everything into consideration that they get to know about their individual situation in the course of the interaction. In contrast, TR demands to determine optimal play entirely from a team perspective that is equally accessible to all members of the team.

The extent of evidence against TR may surprise in the light of the intuitive plausibility that team reasoning seems to have. In fact, in informal conversations with and among subjects after the experiment we often heard arguments that sounded as if they were directly taken from a course in team reasoning: "The best thing we could do was ... so this is what each of us did!"; "My partner did not understand the problem, we should have chosen ... but he did ..." etc.

We have no doubt that TR would earn overwhelming approval by most individuals if introduced as as a normative theory of choice. Moreover, our intuition as well as the anecdotical evidence from discussions about the experiments suggest that there is also some descriptive truth in TR. Team reasoning as a reasoning procedure just seems too familiar to all who get acquainted with the theory.

Our hunch is that the failure of TR in our experiments is not so much due to empirical inadequateness of the team reasoning procedure in principle, but rather a consequence of the specific conditions of choice. Bacharach assumes, that individuals will engage in team reasoning as soon as they take on the team perspective, i.e., as soon as they identify with the team. Sugden adds another necessary condition: individuals need some mutual assurance that others reason in the same way. These conditions still seem to be quite weak and are easily satisfied in our experimental setting. If our observations show that team reasoning as conceptualized in TR does not in fact determine choice to the postulated extent, this may well point to a misapprehension of the conditions that trigger team reasoning rather than to a fundamental misconception in the idea of team reasoning as such.

Still, there might be a more fundamental shortcoming in the background. In TR team reasoning is understood as a procedure of purely individual reasoning. The team is understood as a collective agent, but not as the subject of collective reasoning. So the 'team' in 'team reasoning' points to the object rather than the subject of reasoning. The reasoning process is located entirely in each individual's mind.

The reluctance to ascribe reasoning processes to collectives is possibly grounded in the fact that collectives have no brains or minds beyond the brains and minds of their members. But, of course they also cannot act beyond what can be done by the concert of their members acts; and we still ascribe agency to them. Collective reasoning processes may be hard to define in theory, but they are not mysterious at all. We all know them in practice and we all know how to participate in them. 
Maybe this is the very reason that team reasoning appears so familiar to us.

However, collective reasoning processes in this sense may well require extended communication among the team members - which was carefully precluded in our experiments. How would TR have performed in our experiments, had we allowed partners to discuss things out before playing the game? 


\section{Appendix A: Instructions for ONE-L}

Welcome to the experiment and thank you for your participation!

Please do not talk to other participants of the experiment from now on.

\section{General information on the procedure}

This experiment is conducted to investigate decision making. You can earn money during the experiment. It will be paid to you privately and in cash after the experiment. The entire experiment lasts about 1 hour and consists of 3 parts. At the beginning of each part you will receive detailed instructions. If you have questions after the instructions or during the experiment please raise your hand. One of the experimenters will then answer your question in private. During the experiment you will be asked to make decisions. You will partly interact with other participants, i.e. your own decisions, as well as the decisions of other participants, may determine your earnings. These results from the rules explained in the following. While you make your decisions, you will see a clock running down in the right top corner of your screen. This provides you with some orientation, how much time you should need for your decision. Of course you can also exceed this time, if you need more time for your decision. Especially at the beginning, this may often be the case. Only the information screens, in which no decisions are to be made, will vanish after the time passed out.

\section{Payment}

In each part of the experiment your income is directly stated in Euros. Of part I and part II only one of the parts will be paid out. Which of the two parts will be paid out will be determined randomly and with equal probability by the computer at the end of the experiment (after part III). Your total income is then the sum of your income in part III, and the drawn part (I or II). For your punctual arrival you get $€ 4$ in addition to the income you can receive during the experiment. In the beginning of the experiment you will also receive an endowment of $€ 10$. Your total income is then given by the sum of your credit, the income of Part III, and the part (I or II) which was selected for payment.

\section{Anonymity}

We evaluate all the data of the experiment only in aggregate form and never connect personal information to the data of the experiment. At the end of the experiment you have to sign a receipt for the payment. This only serves for our internal accounting.

\section{Devices}

At your place you will find a pen. Please leave it on the table after the experiment.

Start

In the beginning of the experiment we ask you to choose a color, which will be your personal decision color during the experiment. You will learn for what this color is important in the following instructions. 
On the first screen a list of colors will be displayed. Please mark exactly one of those colors and confirm your choice by clicking the OK-button in the lower part of the screen. All participants choose from the same list of colors. As soon as every participant has chosen her personal decision color the instructions for the first part of the experiment will be distributed.

\section{Part I}

Task

At the beginning of Part I, all participants are divided into teams of two people, which we denote by person A and person B. The allocation is randomly determined by the computer. It will be displayed on the screen if you are person A or person B. You remain member of the same team throughout Part I and Part II. You will not get to know the identity of your team partner at any time of the experiment. Part I consists of five identical rounds. In each round, exactly one decision is to be made. Your decision together with the decision of your team partner will influence your payout as well as your partner's payout. Task In each round, you can choose between option red and option blue. Also, your team partner chooses between option red and blue and you both make your decision at the same time. The income for you and your team partner is as follows:

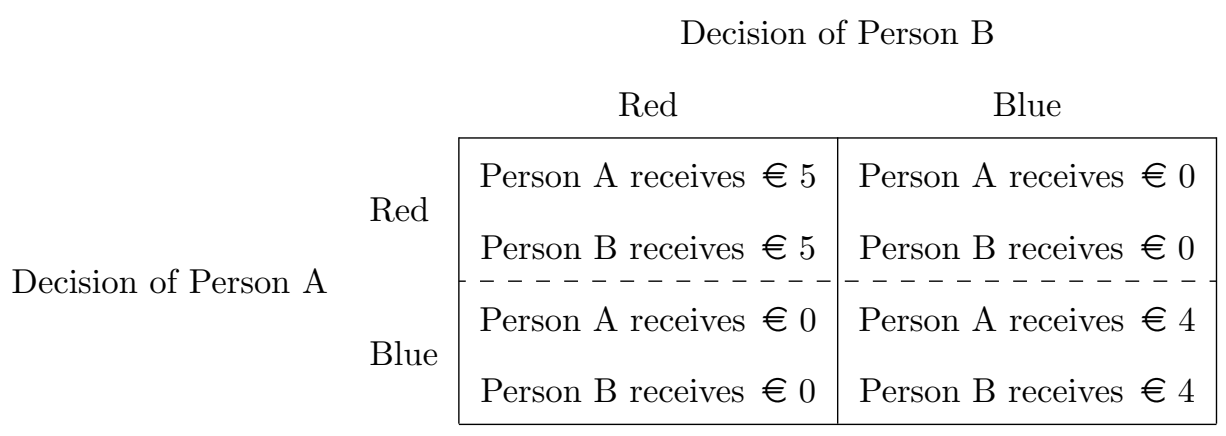

For example: Person A selects red: then you and your team partner both receive $€ 5$ if person $\mathrm{B}$ opts for red as well. If person B decides for blue in this case, you both get $€ 0$. If Person A selects blue, then get you and your team partner both get $€ 0$ if person B chooses red; you both receive $€ 4$ if person $\mathrm{B}$ chooses blue as well.

After both team partners have made their decision in the specific round, you will learn which option your team partner has chosen, and how high your income is in this round, if this round would be selected for payout. Then the next round begins. Once round 5 is completed, Part I ends and the instructions for Part II will be handed out.

If Part I is drawn as the payout relevant part at the end of the experiment (i.e. after the end of Part III), the computer again randomly selects for each team one of the 5 rounds with equal probability $(1 / 5=0.2=$ $20 \%$ ). The amount you have achieved in this selected round, will then be paid out to you in cash. You know at the end of the experiment, whether Part I is payout relevant and if so, what round was selected for you 
and your team partner. Please note: Since you do not know which round is selected, you should devote the same attention to your decisions in each round.

\section{Part II}

In Part II, you are again matched with the same team partner from Part I to form a two-person team. As in Part I you are still person A or person B, and this will again be displayed to you on the screen. Part II consists of one round, that is, you make only one decision which determines, together with the decision of your team partner, your own income and the income of your team partner from this part.

Task

Person A and Person B again decide between option red and option blue and both take their decision simultaneously.

If you are person A, you will have to roll a virtual dice (by the help of the computer) before each person takes her decision. All persons A roll independently of all other persons A in the experiment. The dice's result, can affect the choice options of person A looks as follows. For each person A it holds:

- If Person A rolls a 6, then the decision of that person $\mathrm{A}$ is set to blue.

- If Person A rolls a number other than $6(1,2,3,4$, or 5$)$, then the decision of person A is not affected.

The probability that the decision of person $\mathrm{A}$ is set to blue, is hence $1 / 6$ th. Note that the dice roll only influences the choice of person A and not the decision of person B. Additionally Person B does not learn at any point in time what person $\mathrm{A}$ of her team has thrown. So you do not know if person A can freely choose or if he/she is set to blue.

You will make your decision after every person A has rolled the dice and is informed about its outcome. Your and your team partner's income is as in Part I as follows:

\section{Decision of Person B}

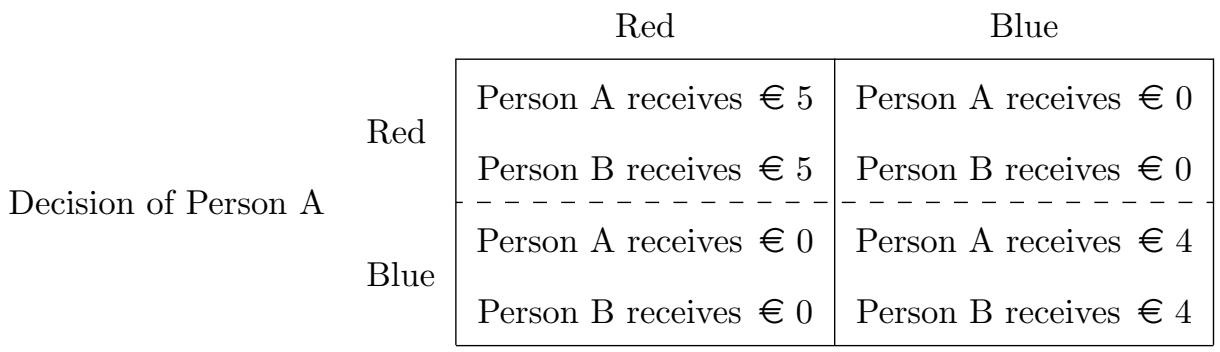

For example: If both people ultimately opted red (or blue), then both receive $€ 5$ (or $€ 4$ ). If one person has red and her team partner has blue (or vice versa), both receive $€ 0$.

After all participants have made their decisions, you are informed about your income from this part of the experiment - in case this part will be drawn at the end as payout relevant. After that ends Part II. 


\section{Part III}

In this part you will only take individual decisions. Hence, your own decisions do not affect the income of other participants and your income is completely independent of the decisions of other participants.

Task

In this part, 16 decision problems are presented to you on the screen. In each of these problems, you can choose between a lottery A and a safe amount of money, which we denote by lottery B. A lottery will remain basically unchanged, only the safe amount of money from lottery B increases with each additional decision problem. Since this safe amount of money is continually increasing, you should as soon as you have decided once for lottery B, do this for all of the following decision problems. An example for such a decision problem is presented in the following table:

Your decisions are only valid if you have made a selection for all of the problems and then clicked on the OK button at the bottom of the screen. Take enough time for your decisions because each can determine your payoff from this part.

After you have taken all the decisions, your earnings from Part III is determined as follows: The computer randomly selects with equal probability one of the 16 decision problems for each participant. If you would have selected lottery $\mathrm{A}$ in this case, the computer will simulate it and you receive the appropriate result. If you have opted for the safe amount of money from lottery B, you get this.

For example: Assume that the computer randomly selects the above decision problem, and you preferred lottery A. Then you either receive $€ 5$ (with probability $5 / 6$ ) or $€ 0$ (with probability $1 / 6$ ) as your payment for this part of the experiment. If you have opted for lottery B, you obtain $€ 3$ with certainty.

After Part III, the experiment ends. We then ask you to complete a few questions about yourself honestly and completely. Once all participants have finished answering these questions, we will call you out one by one in random order based on your subscriber. Your earnings will be paid out privately and in cash.

After the completion of Part III the computer will randomly choose whether Part I or Part II is relevant for your payment. Both parts will be selected with the same probability. 


\section{Appendix B: Supplementary Tables}

\begin{tabular}{|c|c|c|c|c|c|c|}
\hline \multirow[b]{2}{*}{ Treatment } & \multicolumn{2}{|c|}{ Player B } & \multicolumn{2}{|c|}{ Player A } & \multicolumn{2}{|c|}{ Player A \& B } \\
\hline & Risk seeking & Risk averse & Risk seeking & Risk averse & Risk seeking & Risk averse \\
\hline ONE-H & $0.00 \%$ & $37.50 \%$ & $50.00 \%$ & $55.56 \%$ & $33.33 \%$ & $44.00 \%$ \\
\hline$N$ & 2 & 16 & 4 & 9 & 6 & 25 \\
\hline \# red choices & 0 & 6 & 2 & 5 & 2 & 11 \\
\hline$\overline{\mathrm{O}} \overline{\mathrm{N}} \overline{\mathrm{E}}-\overline{\mathrm{L}}$ & $5 \overline{0} . \overline{0} 0 \overline{\%}$ & $\overline{86.6 \overline{7}} \overline{\%}$ & $\overline{100} . \overline{0} \overline{\%} \%$ & $\overline{7} 5.00 \overline{\%}$ & $80.00 \%$ & $\overline{81.48} \%$ \\
\hline$N$ & 2 & 15 & 3 & 12 & 5 & 27 \\
\hline \# red choices & 1 & 13 & 3 & 9 & 4 & 22 \\
\hline TWO-L & $100.00 \%$ & $62.50 \%$ & & $\overline{6} 6.6 \overline{7} \overline{\%}$ & $100.00 \%$ & $64.71 \%$ \\
\hline$N$ & 1 & 8 & 0 & 9 & 1 & 17 \\
\hline \# red choices & 1 & 5 & 0 & 6 & 1 & 11 \\
\hline
\end{tabular}

Notes: enforced decisions again are excluded. A subject is classified as risk averse if her certainty equivalent is smaller than the expected value of respective lottery used in the choice list of Part 3 of the experiment.

Table 2: Frequency of red choices by treatment, role and risk attitude 